\title{
Formation du corps des enseignant-e-s du primaire selon une logique de ségrégation : une approche socio-historique.
}

\section{Céline Delcroix}

\section{OpenEdition}

\section{Journals}

Édition électronique

URL : http://journals.openedition.org/trema/3608

DOI : 10.4000/trema.3608

ISSN : 2107-0997

Éditeur

Faculté d'Éducation de l'université de Montpellier

Édition imprimée

Date de publication : 1 décembre 2016

Pagination : 89-99

ISBN : 979-10-96627-02-8

ISSN : 1167-315X

\section{Référence électronique}

Céline Delcroix, « Formation du corps des enseignant-e-s du primaire selon une logique de

ségrégation : une approche socio-historique. ", Tréma [En ligne], 46 | 2016, mis en ligne le 01 avril 2017, consulté le 01 mai 2019. URL : http://journals.openedition.org/trema/3608 ; DOI : 10.4000/ trema.3608

Ce document a été généré automatiquement le 1 mai 2019.

Trema 


\title{
Formation du corps des enseignant- e-s du primaire selon une logique de ségrégation : une approche socio- historique.
}

\author{
Céline Delcroix
}

Analyser la formation du corps des enseignant-e-s du premier degré permet de mesurer de quelle façon une logique ségrégative a concouru à façonner ce groupe professionnel. Il s'agit en effet de souligner comment un maintien des séparations des sexes et des classes a longtemps opéré dans l'enseignement primaire. Car, à son hégémonie numérique des hommes a répondu une hégémonie massive des femmes dès le XIXe siècle. Et, aujourd'hui, à l'école primaire, les enseignants sont majoritairement des femmes issues des classes favorisées. Ce phénomène est corrélé à une élévation du niveau de recrutement, mais pas seulement. Ainsi, une réelle dialectique des rapports sociaux a construit le corps enseignant au niveau primaire et a modifié ses recrutements toujours en réponse à un refus de mixité sociale et de mixité des sexes. Étudier les processus et les transformations d'un groupe professionnel en termes de féminisation, d'origine sociale et de recrutement permet de mieux saisir sa professionnalité et son identité (Farges, 2011, Lang, 2011). Car, au début du XXe siècle, l'arrivée des femmes dans la profession enseignante est inégale selon les pays (en 1920, aux États-Unis ${ }^{1}$ elles représentent $86 \%$ des enseignants; en Allemagne, en 1911, elles sont 20,9\%; en Belgique, en 1896, elles atteignent $49 \%$, et en Italie, en 1900 , on compte $66 \%$ de femmes enseignantes ${ }^{2}$ ). C'est pourquoi, questionner cette évolution ne peut s'inscrire que dans une agrégation de plusieurs paramètres (conditions économiques, guerres, niveau de recrutement, traditions religieuses, classes mixtes...). Comprendre ce processus comme une construction sociale et non pas comme une simple réponse au fait que les femmes coûtent moins cher et que les hommes délaissent cette profession est un enjeu. L'historiographie anglo-saxonne a largement contribué à éclairer la dimension genrée dans l'histoire de l'éducation (Rogers, 2003a). Et, des lectures croisées de travaux en histoire de l'éducation et en sociologie permettent de souligner l'articulation des rapports sociaux dans la 
construction du corps des enseignants et des enseignantes du primaire. Le genre est entendu dans son acception socio-politique en tant qu'» un système de normes de sexe hiérarchisant, producteur d'inégalités, qui légitime ces inégalités en les naturalisant " Marro (2012, p.74). Il est convoqué ici comme outil de lecture de la structuration de ce groupe professionnel.

2 À partir de textes législatifs, d'enquêtes historiographiques et de recherches en sociologie, cette contribution propose une critique des rapports sociaux au sein de l'organisation enseignante. Cette analyse montre comment une dualité et une séparation des classes et des sexes a construit la profession enseignante au niveau primaire. Il s'agit de souligner la construction sociale du corps enseignant selon une volonté ségrégative des corps et des finalités de l'Éducation en l'abordant dans le temps social et historique. En effet, cette démarche interdisciplinaire permet d'appréhender dans le long et le court terme conjointement l'élaboration d'un groupe professionnel en tant qu'une dialectique du système de genre. Cette complémentarité de deux disciplines, l'Histoire et la sociologie, s'ancre dans notre objet de recherche. De sorte que, cet objet est historique car "les schémas de comportement que notre société inculque à ses membres depuis leur plus tendre enfance, pour en faire une "seconde nature " et que l'organisation du contrôle social maintient en place ne sauraient être expliqués... sans référence à l'Histoire » Elias (1993, p.309). En outre, la posture sociologique permet de mobiliser une nécessaire distanciation pour les différents indicateurs de notre étude. Notre territoire d'enquête commence avec la Troisième République et s'étend jusqu'à nos jours. En effet, l'approche socio-historique, par sa dimension diachronique, permet d'étudier les réseaux de contraintes et de dépendances qui éclairent les transformations de ce groupe professionnel. Et, de questionner pourquoi tant de femmes ont, depuis longtemps, largement investi ce métier, et quelle place est faite aux hommes ? Il s'agit de formaliser comment les rapports sociaux entre hommes et femmes, dans une perspective relationnelle, ont modélisé la formation et le recrutement des enseignants et des enseignantes du premier degré. Nous étudions de quelle façon des logiques ségrégatives et idéologiques concernant la place et le rôle des femmes et des hommes a construit l'enseignement primaire. Nous conduisons notre analyse, à partir du matériau réuni, autour de trois indicateurs de mesure des transformations idéologiques de l'École: l'élévation du niveau de recrutement, l'origine sociale et la sexuation du métier.

3 Ces indicateurs seront développés conjointement selon deux perspectives : tout d'abord pour saisir comment l'enseignement primaire s'est construit à partir d'une logique de séparation des corps dans les formations et les recrutements des enseignant-e-s. Puis dans une seconde partie, nous analyserons comment le genre a modélisé la figure enseignante et définit un stéréotype poursuivant ainsi un ensemble séparé toujours actif.

\section{La constitution d'un enseignement primaire}

4 L'école républicaine sous Jules Ferry, et ce malgré des discours d'inclusion (Dubois, 2007), est le résultat d'une logique ségrégative où deux ensembles scolaires cohabitent : l'école primaire, d'une part, bien souvent située dans les petites communes et dans laquelle se retrouvent les enfants du peuple et les établissements secondaires, d'autre part, installés dans le centre-ville et dans lesquels sont formés les fils de notables (Ben Ayed, 2015, Albertini, 2006). 


\section{I.1. Une logique de séparation}

5 Au début du XIXe siècle, il n'existe pas de réel enseignement primaire homogène en France $^{3}$. Et, si l'enseignement primaire est inégalement réparti dans une opposition nordsud, le contraste s'installe également entre les villes et les campagnes et enfin entre les garçons et les filles. Car, les scolarités en pointillé que connaissent les uns et les unes sont le reflet, d'une part, de la place de l'école pour les familles du peuple et, d'autre part, des visions traditionnelles de la place de la femme qui doit d'abord apprendre la piété et les soins domestiques. Par ailleurs, si déjà beaucoup de classes sont mixtes, compte tenu de la faiblesse des effectifs, cette coéducation ne saurait être que pour les filles du peuple ${ }^{4}$ (Zancarini-Fournel, 2004). Ainsi, bien que la mixité soit refusée dans les textes législatifs sous la Restauration, le Second Empire et la Troisième République, elle fut pratiquée, dans les faits, avec une séparation filles-garçons (avec une cloison d'1m puis d'1,50m, et des sorties décalées pour la récréation) on parle alors de co-instruction (Zancarini-Fournel, 2004). Devant cette réalité scolaire, la loi Pelet (ordonnance royale) du 23 juillet 1836 incite chaque commune à avoir une école primaire de filles. Et, la loi Goblet, article 6 en 1866, établit la part prise par les femmes dans l'enseignement primaire: "L'enseignement est donné par des instituteurs dans les écoles de garçons, par des institutrices dans les écoles de filles, dans les écoles maternelles, dans les écoles ou les classes enfantines et dans les écoles mixtes. Dans les écoles de garçons, des femmes peuvent être admises à enseigner à titre d'adjointes, sous la condition d'être épouse, sœur ou parente en ligne directe du directeur de l'école».

6 En outre, le statut de l'instituteur et de l'institutrice est précaire. Les instituteurs et les institutrices selon l'ordonnance de 1816 sont des laïcs ou des prêtres assermentés qui sont recrutés par le maire après avoir passé un examen « le brevet de capacité » devant un jury issu d'assemblées municipales ce qui met les enseignant-e-s sous la tutelle des pouvoirs locaux. En effet, la modicité de leurs émoluments, à la discrétion de la municipalité ou de l'écolage ${ }^{5}$ les contraint à exercer d'autres services ${ }^{6}$. Mais en 1833 , Guizot alors ministre de l'Instruction publique marque, dans sa Lettre aux Instituteurs, la volonté de l'État de constituer un corps d'enseignant-e-s et d'inspection qui auront comme mission d'unifier la langue et les esprits. Pour se faire, il instaure la réforme du brevet et professionnalise la formation des maîtres et des maîtresses en requérant des aspects pédagogiques à l'examen. Les instituteurs accèdent à une vraie formation et à un vrai traitement puisque cette loi oblige les communes à prendre en charge la maison d'école et le logement de l'instituteur. Reconnu comme une vraie profession, ce groupe professionnel regroupe, en 1833, 32000 instituteurs. L'institutrice, quant à elle, n'a pas le même parcours, son traitement n'est pas fixe. Il faudra attendre 1867 pour qu'elles obtiennent un salaire minimum et qui, toutefois, n'est le minimum de son homologue masculin. De plus, ce n'est qu'en 1879, que la loi Paul Bert oblige chaque département à la création d'écoles normales de filles. Mais l'hostilité envers les écoles normales profite aux congrégations qui disposent d'un "personnel moralement sûr, formé et peu coûteux " (Langlois, 1984, p.324 cité par Condette, 2008). La formation des enseignants et des enseignantes sera laissée à la charge des "cours normaux" qui sont confessionnel, souvent adjacents aux couvents et à l'assentiment du clergé (Mayeur, 2004). Ces cours entendus comme des formations sont, très vite, à destination des maîtres-élèves boursiers et des maîtresses-élèves boursières des Conseils généraux et les préparent à enseigner à la campagne ${ }^{7}$. Peu à peu, les congrégations ont la charge majeure de l'enseignement 
féminin (en raison de la demande de formation des élèves-institutrices, de l'alphabétisation des filles et donc d'écoles qui leur sont destinées, Curtis, 2003).

7 Les enseignantes du primaire sont 22355 en 1840 dont 11984 laïques et 10371 congréganistes (Reboul-Scherrer, 1994). En 1863, 70 \% des institutrices laïques sont des sœurs congréganistes (Rogers, 2007). Les congrégations remplissent largement une mission de service public. En effet, nombre de sœurs congréganistes prennent la place des enseignantes dans les écoles publiques (Curtis, 2003). La modicité du revenu des institutrices laïques et la pression sociale exercée étaient telles que la situation des sœurs pouvait paraître enviable (Mayeur, 2004). Condamnées au célibat, elles devaient mener une vie austère. Cet isolement social difficile dans les campagnes poussera nombre d'entre elles à se réfugier dans les villes sous le statut de sous-maitresse de pensionnat dans l'espoir d'une rémunération et d'une vie moins austère (Rogers, 2003a). Cette pénurie d'enseignantes laïques trouve ainsi son ancrage dans un statut intenable, l'enseignante se trouve déclassée, moins rémunérée que son collègue masculin. Contrainte au célibat, la carrière enseignante n'est pas perçue comme une manière d'accéder à une vie indépendante, c'est un pis-aller pour des femmes seules, soit par le fait d'un veuvage soit à cause de la pauvreté de leur famille qui les prive d'une dot (Cacouault-Bitaud, 2001).

8 À ce terme, les enjeux de l'Éducation sont indissociables de la dualité entre les filles et les garçons, les riches et les pauvres, la ville et la campagne, l'école normale pour les uns et les cours normaux pour les autres boursiers et boursières pour un temps, l'enseignement public laïque et l'enseignement privé des congrégations. Cette construction de la séparation des sexes tant physiquement que symboliquement se perçoit certes dans les finalités de l'éducation mais également dans une détermination de la place et du rôle de chaque sexe.

\section{I.2. Recrutement et Formation}

L'enseignement au niveau primaire est un ordre à part et clos pour les enfants du peuple. Son enseignement ne permet pas la poursuite dans le secondaire. En outre, à cette époque, il y a plus d'élèves dans les écoles congréganistes que dans les écoles laïques (Reboul-Scherrer, 1994). En effet, la loi Falloux permettant aux congrégations d'enseigner avec la lettre d'obédience et non plus avec le brevet de capacité permet d'assurer une continuité des apprentissages quant à la séparation des biens et des personnes, après les mouvements insurrectionnels de 1848 . Toutefois, on ne saurait réduire l'éducation congréganiste à une simple mainmise rétrograde de l'Église sur l'éducation féminine, certes les chiffres attestent d'un monopole, mais ils ne doivent pas occulter les recherches pédagogiques de la maison de Saint Cyr ou de la Légion d'Honneur entre autres qui ont initié des formations pour les femmes de classes populaires comme bourgeoises (Rogers, 2009).

Une volonté laïque s'installe de plus en plus dans le paysage politique souhaitant installer les filles et les femmes dans l'éducation tout en veillant à proposer une " égalité dans la différence » (Rochefort et Zancarini, 2007). En 1880, le nouveau ministre de l'Instruction publique Jules Ferry estime qu'il ne peut y avoir de démocratie sans la diffusion populaire des connaissances. C'est ainsi, que dans sa compréhension d'une réalité éducative "sur les genoux de l'Église ${ }^{8}$, les républicains affirment la nécessité d'une "femme qui appartient à la Science » (Ferry, discours de la salle Molière du 10 avril 1870). Toutefois, si 
les lois Ferry soutiennent considérablement l'éducation des filles et la formation des enseignantes, ce n'est pas dans une logique égalitaire (Rogers, 2009). Les idées républicaines sur l'éducation défendent une profonde différenciation des sexes et des classes (Rochefort et Zancarini, 2007). Pour permettre ce transfert des charges d'éducation de l'Église à l'État, à partir de 1881, les instituteurs et institutrices laïques devront être en possession du brevet de capacité. La mesure était d'importance car il y avait à l'époque 37000 institutrices congréganistes, et seulement 15\% étaient brevetées, la laïcisation était en marche (Prost, 1968). La valence numérique des femmes à l'école primaire n'est pas simplement une réponse à la fuite des hommes vers des emplois plus attirants ou une volonté stricte d'égalité entre les femmes et les hommes (JacquetFrancillon, 2 000). C'est une action de l'état républicain pour laïciser l'éducation féminine et amoindrir le rôle des congrégations religieuses. Dans sa rhétorique anticléricale, la République a utilisé les enseignantes tout en définissant une place précise aux femmes et à leur éducation, tout en leur ouvrant de nouvelles perspectives.

11 Par ailleurs, entre 1880 et 1900, l'exigence d'un brevet élémentaire pour entrer à l'école normale a considérablement modifié le recrutement des futurs enseignants et enseignantes. Car, la faiblesse de la rémunération conjugué à cette élévation du niveau d'entrée a eu comme effet un affaiblissement significatif des candidatures masculines et dans une part plus réduite des candidatures féminines, laissant alors la place à de jeunes suppléantes sans qualification (Chanet, 2000 ; Fergant, 2007). Les jeunes hommes fuient massivement dès cette période ce métier qui ne leur permet pas de s'auto-suffire, il ne permet pas de «nourrir son homme ». Les femmes investissent pour leur part de plus en plus ce métier. Elles se présentent plus nombreuses et sont plus nombreuses à être diplômées que les hommes au brevet et ce à partir de 1873 (Grew et Harrigan, 2002). Toutefois, les écoles normales ne fournissent pas suffisamment d'enseignant-e-s pour couvrir l'ensemble des postes à pourvoir. D'autant que, dans l'enseignement féminin, nombre de candidates formées dans les écoles normales et cours normaux ne s'installent pas dans les campagnes mais se réfugient en ville.

\section{La modélisation de la figure enseignante}

La loi de 1904 interdisant aux congrégations tout enseignement accentuera la féminisation du corps enseignant au niveau primaire, ce que la Grande Guerre ne fera qu'entériner (Chanet, 2007). En effet, la mobilisation des jeunes hommes a comme conséquence le recrutement de nombre d'intérimaires et de suppléantes car les jeunes normaliennes ne suffisent toujours pas pour remplacer les enseignants partis, Jeanperrin $(1915$, p.16).

\section{II.1. La naturalisation d'une profession}

13 De plus, l'exode rural, la baisse de la natalité et le besoin d'économies entraîne de nouvelles expérimentations de la mixité par les classes géminées c'est-à-dire selon un regroupement par âge et non par sexe (Zancarini-Fournel, 2004). Cette réalité scolaire définira le rôle de l'enseignante « Le cours préparatoire, le cours élémentaire sont assurés par une institutrice mariée, mère de famille le plus souvent, comprenant la nature et l'âme de l'enfant, sachant diriger ses premiers pas vers la voie du savoir, sachant l'aimer et lui inculquer les premiers éléments de l'instruction et de la morale.» (Anatole de 
Monzie, ministre de l'Éducation en 1933). L'enseignante n'est pas une professionnelle c'est une mère de famille qui déploie des qualités personnelles dans la sphère publique. Sa posture professionnelle est naturalisée la renvoyant à sa nature de mère et d'épouse potentielle. De sorte que la loi Roustan de 1921 conjugue deux perspectives : nommer des couples mariés dans les écoles géminées et également faciliter le rapprochement de conjoints. Ainsi, l'accroissement des candidatures féminines trouve un effet dans cette loi conjugué au fait que, en 1919, les traitements des institutrices sont alignés sur ceux des instituteurs. C'est ainsi que la médiocrité des conditions matérielles des enseignant-e-s du primaire fait naître un nouveau modèle : épouser un ou une collègue dans l'idée que " deux traitements insuffisants permettent, de l'avis général, de vivre convenablement en ménage » Mayeur (2004). Ainsi, une évolution rapide se produit et en regardant l'étude menée par Jacques Ozouf, 1966 sur les enseignant-e-s de la Manche, on constate que dans ce département, on passe de $18 \%$ d'institutrices mariées en 1897 à $27 \%$ en 1903 et $56 \%$ en 1922. Si on l'extrapole, on peut dire que très tôt dès 1920 " le modèle de la femme mariée supplante celui de la femme célibataire » Mosconi (1997, p.128). L'hégémonie numérique se poursuit et, dès cette époque, 1911-12, les institutrices exerçant dans le primaire et le primaire supérieur forment déjà $58,2 \%$ du corps enseignant, la proportion atteint $67,4 \%$ en 1939-40 (Cacouault-Bitaud et Zaïdman, 1994). Toutefois, malgré la précarité économique pour nombre d'instituteurs et d'institutrices, la mission d'instruire et d'éduquer le peuple dont la République les a investis, leur confère un prestige social. De plus, les enseignants et les enseignantes ont largement participé au "désarmement moral » après la Grande Guerre, leur rôle pacifiste et patriotique fut majeur dans la reconstruction (Siegel, 2004). Cette conception essentialiste de la profession enseignante va marquer les compétences professionnelles mais aussi les carrières enseignantes. Ainsi, dans une logique de substitution de la mère, les femmes transféreront des compétences «maternelles » dans leur profession, et les hommes prendront la figure de l'autorité (Martino, 2008 ; Zaïdman, 1996). À ce titre, l'institution a réservé des postes aux femmes et n'en a pas donné l'accès aux hommes. Ainsi jusqu'en 1972, l'encadrement des écoles maternelles étaient réservé aux inspectrices. De même, seules les femmes pouvaient enseigner en maternelle jusqu'en 1977. Et enfin, le recrutement du personnel enseignant dans les écoles normales, bien que leurs formations soient mixtes, est soumis aux quotas jusqu'en 1984 : 52,9\% pour les femmes et 47,1\% pour les hommes (Hirschhorn, 1993 cité par Jaboin, 2008).

\section{II.2. Une élévation continue du niveau de recrutement}

14 Ainsi, ces « fils ou filles du peuple » symbolisent la mobilité sociale et la promotion liée aux études pour les enseignant-e-s nés avant 1914, ils sont souvent issus de milieux économiquement pauvres et ils ont un goût vif pour l'instruction, Ozouf (2001, p. 46). Le gouvernement de Vichy (loi du 18 septembre 1940) exige le baccalauréat pour devenir enseignant-e après trois années de lycées ${ }^{9}$ et rend obligatoire les cours d'enseignement ménager pour les filles et les femmes afin d'entretenir «l'éternel féminin » (MuelDreyfus, 1996). Après la Seconde guerre mondiale, les corps enseignants du premier et du second degré vont connaitre une extension numérique considérable. La féminisation du corps enseignant au niveau primaire s'accentue encore. En effet en 1962, «le taux de féminisation est de 68\% » (Berger, 1979). 
15 Le sens commun véhicule l'idée selon laquelle le métier d'enseignante est un métier «féminin » puisqu'il permet d'articuler les vies professionnelle et familiale. Toutefois, si l'enseignement a été représenté comme un métier typiquement féminin en tant que prolongement du rôle de la femme comme mère et éducatrice. Il a également permis aux femmes de s'instruire et d'embrasser une profession «libérale " avec des salaires qui, bien que modestes, garantissaient cependant l'indépendance économique, avec des responsabilités pédagogiques, des possibilités de carrières promotionnelles, mais aussi l'opportunité de prendre part à la vie citoyenne par le biais d'engagements syndicaux, associatifs voire politiques (Cacouault-Bitaud, 2007). En outre, les transformations dans le mode de recrutement de la fin de troisième à un niveau bac puis à un niveau DEUG apparaissent comme un facteur clef pour expliquer la crise des écoles normales dans les années 1980 et l'effondrement brutal des candidatures (Charles et Clément, 1997). Tout d'abord cette modification de recrutement va donner lieu un renversement de la tendance qui amenait à l'École normale davantage d'élèves venant des classes populaires que des autres groupes sociaux. Les enseignant-e-s sont alors issu-e-s principalement des cadres moyens et supérieurs, et des professions libérales, Berger (1979, p.25), un lent « embourgeoisement» de ce groupe professionnel est en marche. Cependant, la politique de massification de l'enseignement et d'allongement des études va permettre de répondre à la demande. À partir de 1985, la très forte augmentation du nombre des bacheliers et par suite des diplômé-e-s de l'enseignement supérieur, va contribuer à l'accroissement progressif du nombre de candidats et de candidates. Mais la création des IUFM, en 1989, et le recrutement sur la base d'une licence marque une période d'ajustement et se caractérise par une forte croissance des postes offerts qui correspond à la compensation de nombreux départs à la retraite d'enseignant-e-s recruté-e-s dans les années 1950, (Charles et Clément, 1997). Ces divers modes de recrutement vont modifier la composition sociale du public des enseignant-e-s. Frédéric Charles et Jean-Paul Clément, 1997, précisent l'origine sociale respective des enseignant-e-s qui sont issues des catégories supérieures (à $30.5 \%$ ), moyennes (à $50.8 \%$ ) et populaires (à $18.4 \%$ ). Ces choix politiques d'un recrutement à un niveau de formation plus élevé a conduit les étudiants des classes populaires à renoncer à l'entrée dans le métier et a de la sorte favorisé une élévation du niveau social des enseignant-e-s du primaire (Charles et Cibois, 2010). Cette idée fait écho aux études locales, en Alsace de Frédéric Charles et Jean Paul clément, 1997, en Poitou Charente de Sébastien Ramé, 1999, à Versailles de Roger Roussel, 2001 et en Bretagne d' Yveline Jaboin, 2007, qui observent que chez les parents des enseignant-e-s, les catégories supérieures et moyennes sont surreprésentées par rapport aux ouvriers pour les pères et aux employées pour les mères. Ainsi, à l'image préconstruite antérieurement d'une profession identifiée à une voie de promotion sociale des enfants de familles modestes, s'oppose le constat évident, que la logique de recrutement de l'institution scolaire a consacré l'héritage et non la promotion sociale. L'investissement des classes dominantes dans le professorat s'explique par des stratégies d'investissement scolaire ou par un déclassement. En somme, le professorat des écoles est exercé par des enseignant-e-s issu-e-s de classe qui l'avaient « longtemps ignoré voire méprisé » Charles (1997, p.49). En 2008, le niveau de diplôme est porté au master pour accéder au métier de l'enseignement dans le premier comme dans le second degré. Cette décision de formation du personnel enseignant est à placer dans la continuité historique d'une évolution et d'une élévation du niveau universitaire des professeur-e-s tant au niveau de la politique publique (d'une réglementation européenne) qu'au niveau social (quant au profil des candidat-e-s) (Larivain, 2006). La masterisation a comme effet de pérenniser l'éviction des 
étudiant-e-s issu-e-s des classes populaires (Rapport IGEN- Juillet 2013). C'est pourquoi, l'élévation continue du niveau de recrutement des enseignant-e-s du premier degré a pour effet d'agir sur la durée comme une barrière pour certaines classes sociales, les écartant au fil du temps de plus en plus significativement et amenant d'autres classes sociales à investir ce métier.

\section{Discussion conclusive}

L'histoire de la mixité, sociale et des sexes, est longue et sinueuse tant du point de vue des élèves que des personnels. Et, analyser la formation du corps des enseignant-e-s du primaire permet de mettre en lumière la façon dont la profession est devenue à hégémonie numérique des femmes et s'est construite comme un métier de femmes dans une logique de ségrégation. Aujourd'hui, le corps enseignant est composé de $82,6 \%$ de femmes et 17,4\% d'hommes (INSEE, 2016) dans le primaire. Pour les diplômées de l'enseignement supérieur, potentielles mères de famille, l'enseignement «semble aujourd'hui suivre un stéréotype social largement diffusé comme une profession accessible et souhaitable » (Jaboin, 2003). Dans le prolongement de ce phénomène, il est également important de montrer de quelle manière l'élévation du niveau de recrutement des futur-e-s enseignant-e-s n'a eu de cesse d'augmenter, agissant comme un filtre social en modifiant significativement l'origine sociale des enseignant-e-s qui entrent dans la profession. Parallèlement, la désaffection des hommes pour le métier, «le péril primaire ", apparaît significativement dès 1900 (Grandière, 2006 cité par Condette, 2008) selon une agrégation de paramètres : la fin de l'exemption militaire, la difficulté à obtenir le concours ainsi que l'attrait croissant « pour des professions commerciales, industrielles et administratives " plus rémunératrices (Condette, 2008, p.136). À cela s'ajoute que l'enseignement primaire est pensé, pour les femmes10, comme le transfert de charges domestiques et familiales en compétences professionnelles (Jarty, 2009). L'école primaire a été conçue comme un ordre à part et à destination des enfants du peuple. Il s'agissait selon les instructions de 1882 de transmettre à ces élèves tout le savoir pratique pour leur vie future. Comme les salles d'asile étaient un accueil des enfants pauvres et notamment des familles ouvrières obligées de travailler. La séparation des classes, des sexes et des savoirs était opérante. Or, aujourd'hui, malgré la mise en place de la mixité des sexes (décrets Haby), de textes et de conventions sur l'égalité des sexes (IGEN 2013, MEN 2006), et avec la démocratisation scolaire, la France reste parmi les pays de l'OCDE où la mixité sociale est la plus faible chez les élèves. Ainsi, l'École française très attachée formellement au principe de laïcité et de mixité propose toutefois une égalité dans la différence. Cette séparation des classes et des sexes à l'école pose des questions de professionnalité pour le corps enseignant. Que penser d'une école «maternelle» où encore pratiquement exclusivement des femmes exercent? Quelle place est donnée aux hommes qui souhaitent y enseigner? Cette construction socio-sexuée du corps enseignant n'est donc pas sans effet sur les personnes, notamment au niveau des enjeux en termes de féminité et de masculinité mais également au regard de la professionnalité tant pour les personnels que pour les élèves qui évoluent dans un ensemble séparé actif. 


\section{BIBLIOGRAPHIE}

Albertini, P. (2014) L'école en France. Du XIXe siècle à nos jours. Paris : Hachette Supérieur.

Ben Ayed, C. (2015) La mixité sociale à l'école : tensions, enjeux, perspectives. Paris : Armand Colin.

Berger, I. (1979) Les instituteurs d'une génération à l'autre. Paris : PUF.

Cacouault-Bitaud, M. (2007) Professeurs...mais femmes, carrières et vies privées de enseignantes du secondaire au XXe siècle. Paris : La découverte.

Cacouault-Bitaud, M. (2001) « La féminisation d'une profession est-elle le signe d'une baisse de prestige ? » Travail, genre et sociétés, n5, p.93-115.

Cacouault, M. et Zaïdman C. (1994) « les politiques nationales concernant la formation des femmes : le cas de la France » in Femmes et éducation : politiques nationales et variations internationales, sous la direction de Margaret B. Suterland et Claudine Baudoux, les cahiers du Labras : Université de Laval, 1994.

Cacouault-Bitaud, M. (1995) « féminisation et masculinisation des professions : approche historiques et comparatives » les cahiers du MAGE, $\mathrm{n}^{\circ} 1$.

Chanet, J. F. (2000) « Vocation et traitement. Réflexions sur la "nature sociale" du métier d'instituteur dans la France de la IIIe République ». Revue d'histoire moderne et contemporaine, 47-3, p.581-603.

Chanet, J.F. (2007) « Des institutrices pour les garçons : la féminisation de l'enseignement primaire en France des années 1880 aux années 1920 ». Histoire de l'éducation, INRP, p.171-193.

Charles, F. et Cibois P. (2010) «L'évolution de l'origine sociale des enseignants du primaire sur la longue durée : retour sur une question controversée ", Sociétés Contemporaines, 77, p.31-55.

Charles, F. et Clément J. P. (1997) Comment devient-on enseignant ? l'IUFM et ses publics. Strasbourg : Presses universitaires de Strasbourg.

Condette, J.F. ( 2008) Histoire de la formation des enseignants en France. Paris : L'Harmattan.

Curtis, S. (2003) L'enseignement au temps des congrégations : Le diocèse de Lyon (1801-1905), Lyon : PUL. Dubois, P. (2007) «Figures de l'école juste et politique scolaire dans les années fondatrices de la Troisième république » Revue Française de pédagogie, n¹59, 2, p.13-22.

Elias, N. (1993) Engagement et distanciation. Paris : Fayard.

Farges, G. (2011) « Le statut social des enseignants français », Revue européenne des sciences sociales, 49-1, p.157-178.

Fergan,t A. (2007) Les instituteurs de Seine-et-Oise (1880-1914), thèse de doctorat d'histoire, Université Paris-XIII.

Grandière, M.(2006) La formation des maîtres en France (1792-1914). Lyon : INRP.

Grew, R. et Harriman P.J.(2002) L'école primaire en France au XIXe siècle : essai d'histoire quantitative, traduit de l'anglais par Marie-Pierre Gaviano, Paris, Éd. de l'EHESS.

Hirschhorn, M. (1993) L’ère des enseignants. Paris : PUF. 
INSEE, 2016. Enseignants du public et du privé par corps en 2015, janvier 2016, http:// www.insee.fr/fr/themes/tableau.asp?reg_id=0\&ref_id=nattef07115 [consulté le 10/05/2016].

Jaboin, Y. (2008) « La construction de l'identité professionnelle masculine dans un secteur en voie de mixité : le cas des hommes enseignant à l'école maternelle » In Guichard-Claudic (Yvonne), Kergoat (Danièle), Vilbrod (Alain) (dir.) L'inversion du genre, quand les métiers masculins se conjuguent au féminin...et réciproquement, Rennes : PUR.

Jaboin, Y. (2007) «Les déterminants sociaux et scolaires des carrières enseignantes : le cas des professeurs des écoles stagiaires à l'IUFM de Bretagne ». Carrefours de l'Education, n²3, p.119-135.

Jaboin, Y. (2003) Le prof dans tous ses états féminin ou masculin, public ou privé. Paris : Fabert.

Jacquet-Francillon, F. (2000) «La féminisation de l'enseignement, une histoire de femmes ?» Mélanges de la Maison de Saint-Éxupéry, Lille.

Jarty, J. 2009, « Les usages de la flexibilité temporelle chez les enseignantes du secondaire », Temporalités [En ligne], 9.

Jeanperrin, A. (1915), dans son rapport 1914-1915, BD Jura, supplément au nº 520.

Lang,V. (2011) « La question de la reconnaissance des maîtres de l'enseignement primaire public en France : approches socio-historiques » in Anne Jorro et J.M. De Ketele, La professionnalité émergente : quelle reconnaissance. Bruxelles : De Boeck.

Larivain, C. (2006) «Les enseignants des écoles publiques et la formation », Dossiers du ministère de l'Éducation nationale, $\mathrm{n}^{\circ} 176$.

Marro, C. (2012) « Dépendance-Indépendance à l'égard du genre. Penser l'égalité des sexes audelà de LA différence » Recherche et Formation, 69, p.65-80.

Martino, W, (2008) « The lure of hegemonic masculinity: investigating the dynamics of gender relations of two male elementary school teachers' lives » International Journal of Qualitatives Studies in Education, 21-6, p.575-603.

Mayeur, F. (2004) Histoire générale de l'enseignement et de l'éducation en France, 1789-1930, Paris : Tempus.

Mayeur, F. (1979) l'Éducation des filles en France au XIXe siècle. Paris : Hachette.

Ministère de l'Éducation nationale (2006). Convention interministérielle pour l'égalité des chances entre les filles et les garçons, les femmes et les hommes dans le système éducation. Paris : MEN.

Mosconi, N. (1997), Femmes et savoir : la société, l'école et la division sexuelle des savoirs, Paris :

L'Harmattan.

Muel-Dreyfus, F.(1996), Vichy et l'éternel féminin. Paris : Seuil.

Ozouf, J.(1966) « Les instituteurs de la Manche et leurs associations au début du siècle », revue d'histoire contemporaine, p.95-114.

Ozouf, J. et M. (2001) La république des instituteurs, Paris : Points Histoire.

Prost, A. (1968) Histoire de l'enseignement en France 1800-1967. Paris : Colin.

Ramé, S. (1999), L'insertion professionnelle et sociale des nouveaux enseignants. Paris : l'Harmattan.

Reboul-Scherrer, F. (1994) Les premiers instituteurs 1833-1882. Paris : Hachette.

Rochefort, F. et Zancarini, M. (2007) « Laïcité, égalité des sexes et mixité scolaire » Spirale n³9, p.39-51. 
Rogers, R. (2009) « revoir l'histoire de l'éducation sous l'angle du genre : nouvelles perspectives » in Bodinier B., Guest M., Lemonnier-Delpy M-F, Pasteur P. « Former, se former, être formée au féminin » Genre et Éducation. Rouen, PURH.

Rogers, R. (2007) « Les enseignantes religieuses et laïques au XIXe siècle: vocation ou activité professionnelle » in Causer J.Y., Pfefferkorn R., Woehl B., Métiers, genre et identités professionnelles. Paris : L'Harmattan, 2007, p.33-50.

Rogers, R. (2003a) « La sous-maîtresse française au XIXe siècle: domestique ou enseignante stagiaire ? ». Histoire de l'éducation, n98, p.37-60.

Rogers, R. (2003b) «État des lieux de la mixité. Historiographies comparées en Europe ». Clio. Histoire, femmes et sociétés, 18, p.177-202.

Roussel, R. (2001), « une petite noblesse d'État ou des professionnels compétents ? » in Ramé S. Insertions et évolutions dans le milieu enseignant. Paris : l'Harmattan.

Siegel, M. L. (2004) The Moral Disarmament of France. Education, Pacifism, and Patriotism, 1914-1940. Cambridge, Cambridge University Press.

Zaïdman, C. (1996) La mixité à l'école primaire. Paris : L'Harmattan.

Zancarini-Fournel, M.(2004) « Coéducation, gémination, co-instruction, mixité : débats dans l'Éducation nationale (1882-1976) » in Rogers R.(dir.) La mixité dans l'éducation. Enjeux passés et présents. Lyon : ENS Éditions, p.25-32.

\section{NOTES}

1. Brumberg J. (1983) «The Feminization of Teaching: "Romantic Sexism » and American Protestant Denominationalism ». History of Education Quaterly, 23.

2. Albisetti J. (1993) « The Feminization of Teaching in Nineteenth Century: a comparative perspective ». History of Education, 22.

3. L'enquête de Maggiolo L. réalisée en 1877 et malgré ses biais montre une France coupée en deux de Saint Malo à Genève avec au nord une scolarisation importante et au sud une insuffisance générale.

4. Compayré G.(1879) Histoire critique des doctrines de l'éducation en France. Paris : Hachette, précise : «Pour les classes inférieures de la société et sur les bancs de l'école primaire et jusqu’à un certain âge la promiscuité ne nous déplaît pas. Mais quand il s'agit d'une culture haute et plus raffinée, quand les élèves appartiennent à un rang plus élevé, il nous semble impossible de donner le jeune homme pour camarade à la jeune fille ".

5. Somme demandée à chaque père de famille selon le niveau d'instruction de son enfant.

6. On trouve des secrétaires de mairie, bedeau du curé, barbiers, aubergistes, géomètres, greffiers, cultivateurs...In Prost A. (1968) Histoire de l'enseignement en France 1800-1967. Paris : Colin.

7. Les cours de travaux d'aiguille n'abordent pas la broderie, à destination des jeunes filles bourgeoises, dans les cours normaux mais le rapieç̧age et le remmaillage des bas et des chaussettes in Mayeur (2004).

8. Monseigneur Dupanloup.

9. L'échec du système est immédiat: à la veille de l'entrée à l'Institut de formation professionnelle, « la plupart des promotions fondent désespérément on compte dans certains cas jusqu'à 8/10èmes d'abandons ", Prost A. (1968), op.cit. 


\section{RÉSUMÉS}

Analyser la formation $\mathrm{du}$ corps des enseignant-e-s du primaire, dans une perspective diachronique, permet de saisir de quelle façon son identité professionnelle s'est façonnée et de mesurer les modifications d'un groupe professionnel. Les transformations rencontrées en termes de sexuation, de niveau de recrutement et d'origine sociale élaborent un ensemble séparé pour les enseignant-e-s du primaire et définissent une dialectique des rapports sociaux de sexe au sein de l'École.

Analyzing body training of primary school teachers, in a diachronic perspective, captures how his professional identity is shaped and measure the changes of a professional group. The transformations experienced in terms of gendering, level of recruitment and social origin develop a separate set for primary school teachers and define a dialectic of gender relations within the school.

\section{INDEX}

Mots-clés : enseignement, primaire, féminisation, origine sociale, recrutement

Keywords : primary education, feminization, socially origin recruitment

\section{AUTEUR}

\section{CÉLINE DELCROIX}

ESPE de Créteil - Université Paris Est Créteil (UPEC), Observatoire Universitaire International Éducation et Prévention (OUIEP), Laboratoire Interdisciplinaire de Recherche sur les Transformations des pratiques Éducatives et des pratiques Sociales (LIRTES) 